\title{
Extracranial Complications of Traumatic Brain Injury: Pathophysiology-A Review
}

\author{
Parameswara Gundappa ${ }^{1}$ \\ ${ }^{1}$ Department of Anaesthesiology, Manipal Hospital, Bengaluru, \\ Karnataka, India
}

J Neuroanaesthesiol Crit Care 2019;6:200-212

\begin{abstract}
Address for correspondence Parameswara Gundappa, MD, FICA, Department of Anaesthesiology, Manipal Hospital, 98, HAL Airport Road, Bengaluru 560017, Karnataka, India (e-mail: paramgundappa@gmail.com).
\end{abstract}

\author{
Abstract \\ Keywords \\ - extra cranial \\ complications \\ - traumatic brain injury \\ - neurogenic pulmo- \\ nary edema \\ - sepsis \\ - coagulation \\ abnormalities \\ - neurogenic immune \\ response \\ - peripheral immune \\ suppression \\ - neurogenic stunned \\ myocardium
}

Moderate to severe traumatic brain injury is often associated with several extracranial organ complications, which increase the morbidity and mortality. Respiratory complications such as acute lung injury, pneumonia, and acute respiratory distress syndrome (ARDS) occur most commonly, but neurogenic pulmonary edema can be life threatening. Cardiovascular complications occur frequently. However, arrhythmia, cardiogenic shock, and neurogenic stunned myocardium, though occur infrequently, can be life threatening. Coagulation abnormalities and sepsis constitute serious complications that may result in multiorgan failure. These constitute independent risk factors for mortality. Endocrine abnormalities, gastrointestinal disruptions, and other complications occur less commonly. These extracranial complications develop as a result of altered neurogenic immune response, both central and peripheral responses. Brain tissue injury releases both proinflammatory mediators taking part in tissue reparative process and anti-inflammatory cytokines that propagate inflammation. In addition, release of massive amount of catecholamines after head injury results in proliferation of myeloid depressor cells in the circulation, release of reactive oxygen species, and release of immature neutrophils into the circulation. These anti-inflammatory mediators by complex mechanisms inhibit and decrease the number of $\mathrm{T}$ cells and cause apoptosis. This results in decreased production and release of cytokines and reduced lymphocyte activated killer cell cytotoxicity, resulting in impaired hypersensitivity reaction. Finally, the complex interplay of various factors leads to suppression of peripheral immune response and susceptibility for infection and sepsis, and causes extracranial organ system failure with increased morbidity and mortality.

\section{Introduction}

India has a very high incidence of road accidents. Every year more than 1.5 lakh people get injured in road traffic accidents, with a case fatality rate of $18 \%$. Shekhar et al $^{1}$ in their study reported that about $60 \%$ of road accidents in India result in traumatic brain injury (TBI), with an overall mortality rate of $29 \%{ }^{1}$ In a recent study by Kamal et al, ${ }^{2}$ out of 1,527 patients with moderate to severe TBI admitted to All India Institute of Medical Sciences, New Delhi, mortality was $34 \%$ and remaining $67 \%$ had an unfavorable outcome at 6 months. ${ }^{2}$ Mortality due to TBI is often related to direct injury to the brain and

received

November 6, 2018 accepted after revision

April 15, 2019

published online

July 14, 2019 its consequences of increased intracranial pressure (ICP), contusions, hemorrhages, or diffuse injuries. Nevertheless, a significant number of patients with TBI develop systemic complications such as of respiratory, cardiac, renal, and other systems. These extracranial complications (ECCs) increase the morbidity as well as mortality of TBI.

Several studies have shown that TBI results in systemic extracranial injuries that may complicate brain injury. ${ }^{2-4}$ It is interesting and imperative to understand why and how brain injury causes extracranial systemic involvement. This review discusses the various ECCs due to TBI and pathophysiology involved in the development of these complications.
Copyright $\odot 2019$ Indian Society of Neuroanaesthesiology and Critical Care

License terms

10.1055/s-0039-1692883 ISSN 2348-0548. 
Table 1 Extracranial complications related to brain injury

\begin{tabular}{|c|c|}
\hline $\begin{array}{l}\text { Respiratory } \\
\text { complications }\end{array}$ & $\begin{array}{l}\text { Acute lung injury } \\
\text { Acute respiratory distress syndrome } \\
\text { Pulmonary edema } \\
\text { Pneumonia } \\
\text { Atelectasis } \\
\text { Aspiration pneumonitis }\end{array}$ \\
\hline $\begin{array}{l}\text { Cardiovascular } \\
\text { complications }\end{array}$ & $\begin{array}{l}\text { Hypotension } \\
\text { Hypertension } \\
\text { Arrhythmias } \\
\text { Shock } \\
\text { Neurogenic stunned myocardium } \\
\text { (Takotsubo's cardiomyopathy) }\end{array}$ \\
\hline Renal complications & Acute kidney injury \\
\hline $\begin{array}{l}\text { Gastrointestinal } \\
\text { complications }\end{array}$ & $\begin{array}{l}\text { Cushing's ulcers } \\
\text { lleus }\end{array}$ \\
\hline $\begin{array}{l}\text { Hematologic } \\
\text { complications }\end{array}$ & $\begin{array}{l}\text { Platelet dysfunction } \\
\text { Coagulopathy }\end{array}$ \\
\hline Infections & Sepsis and septic shock \\
\hline $\begin{array}{l}\text { Endocrine } \\
\text { complications }\end{array}$ & $\begin{array}{l}\text { Electrolyte imbalances } \\
\text { Syndrome of inappropriate antidi- } \\
\text { uretic hormone secretion } \\
\text { Cerebral salt-wasting syndrome } \\
\text { Hypopituitarism } \\
\text { Hypoadrenalism } \\
\text { Hypothyroidism }\end{array}$ \\
\hline $\begin{array}{l}\text { Metabolic } \\
\text { derangements }\end{array}$ & $\begin{array}{l}\text { Hypoglycemia } \\
\text { Hyperglycemia } \\
\text { Hyperthermia }\end{array}$ \\
\hline
\end{tabular}

Treatment of these complications is beyond the scope of this review. Various systemic complications reported due to TBI are given in - Table $\mathbf{1}$.

One of the earliest clinical studies on ECC was reported by Piek et al in 1992. ${ }^{3}$ In their analysis of 734 patient records from Traumatic Data Bank, there were 1,709 ECCs. In a similar retrospective study by Schirmer-Mikalsen et al, ${ }^{4} 133$ case records of patients with only TBI were studied. Pneumonia was diagnosed in $71 \%$ and acute lung injury (ALI)/adult respiratory distress syndrome (ARDS) in 26\% patients. Other complications such as severe sepsis (6\%), renal failure (1.5\%), and a coagulation disorder (6\%) were the major ECCs. Age, Glasgow coma scale (GCS), hypotension during the first day of treatment, elevated blood sugar, and low albumin predicted an unfavorable outcome. Corral et $\mathrm{al},{ }^{5}$ in their retrospective observational cohort study of TBI, analyzed data of 224 patients admitted to intensive care unit (ICU), with GCS $<9$ for neurological and non-neurological complications and their impact on mortality.

Even though the reported incidence of different complications varies between these above studies, the major complications reported were sepsis (10-75\%) and septic shock (6-8\%), followed by respiratory infections, including pneumonia (28-68\%), respiratory failure (41\%), ALI, and ARDS (9-28\%). Cardiovascular complications were hypotension (20-44\%), hypertension (12\%), and requirement of vasopressors (70\%). Coagulopathy (6-27\%), electrolyte disturbances (21-59\%), and acute renal failure (1.5-8\%) were other systemic complications. Statistically significant mortality was reported due to ARDS (90\%), hypoxia (69\%), septic shock (85\%), bleeding complications (50\%), acute kidney injury (AKI) (76\%), and non-neurological surgery (21\%). Respiratory and cardiovascular complications were the most common early complications occurring within 3 to 4 days, with pneumonia (40.6\%) and septicemia (10\%) occurring in the next 5 to 10 days. Gastrointestinal, renal, and hepatic complications were late complications occurring beyond second week. Other less common complications were low serum albumin (31\%), hyperthermia (24\%), and anemia (22\%). Whereas GCS 3 to 5 and intracranial hypertension were neurological variables associated with mortality directly due to TBI, age, pulmonary complications, coagulopathy, AKI, and septicemia were found to be independent predictors of mortality. Non-neurological surgeries also increased the mortality in ICU. Other non-neurological complications increased the length of stay and morbidity in ICU but did not increase the mortality. The overall mortality related to extracranial organ dysfunction varied between 26 and $35 \%$.

In a prospective observational cohort study, Zygun et $\mathrm{al}^{6-8}$ combined trauma service data with ICU data, to study the incidence of non-neurological organ dysfunction in patients admitted to ICU. Maximum modified multiple organ dysfunction (MOD) score of $\geq 3$ was considered as having organ dysfunction. Nearly $10 \%$ of patients under trauma service data had severe head injury admitted to ICU, and $89 \%$ of these patients developed ECC of at least one organ. Respiratory dysfunction was the most common complication (81\%) with respiratory failure (23\%) followed by cardiovascular dysfunction (52\%) with cardiac failure (18\%). Coagulation abnormalities (17\%) with coagulation failure (4\%) and renal dysfunction (8\%) were less common complications. The overall mortality in their study was $32 \%$. Mortality was independently associated with MOD score and worst GCS. Extracranial injuries with high Abbreviated Injury Score (AIS) were also associated with higher ICU mortality, emphasizing the importance of extracranial injuries in worsening TBI mortality. However, the timing of organ dysfunction did not influence the mortality. Sequential Organ Failure Assessment (SOFA) scoring system was found to be superior to MOD score with respect to hospital mortality in relation to non-neurological complications. ${ }^{9}$

These and several other studies have shown that systemic organ dysfunction can occur with head injury even in the absence of systemic non-neurological injury. These extracranial systemic dysfunctions can occur either during acute or recovery phase and even in later period. No correlation between the severity of the TBI and systemic manifestations has been shown, with ECC occurring even in mild head injury.

\section{Pathophysiology of Extracranial Non-neurological Systemic Complications}

Severe head injury results in (1) primary brain injury directly related to primary impact and (2) secondary brain injury as a result of secondary effects of increased ICP and decreased cerebral perfusion pressure. In addition, TBI may result in many extracranial systemic injuries. The factors responsible 
for development of this non-neurological systemic dysfunction are result of a complex interplay of multiple factors involving autonomic nervous system, neuroendocrine dysfunction, neuronal immune and inflammatory responses, systemic immune response, and biochemical cascades. ${ }^{10}$

\section{Neural Immune Response to Injury}

Cerebral injury has been shown to produce depression of systemic immune response. Various complex mechanisms have been proposed for this immunity depression due to interplay of many vasoactive mediators and activity of immunity-related cells.

The immune modulation of the brain to cellular injury includes release of pro- and anti-inflammatory mediators. Cytokines include chemokines, interferons, interleukins (IL), lymphokines, tumor necrosis factor (TNF). The proinflammatory cytokines include mediators such as interleukins (IL-1, IL-6, and TNF- $\alpha$, IL-18), and anti-inflammatory cytokines include IL-10, and TGF- $\beta$ (transforming growth factor- $\beta$ ). Chemokines that include IL- 8 are chemotactic in nature and attract leukocytic and monocytic chemotactic proteins. Brain injury may result in cellular death and release of nuclear and mitochondrial damage-associated molecular patterns (DAMPs) such as mDNA, $\mathrm{N}$-formyl peptides into the circulation through disrupted blood--brain barrier. DAMP molecules can initiate and propagate noninfectious inflammation.

Head injury at the time of crash is associated with severe sympathetic stimulation associated with massive catecholamine release. ${ }^{11,12}$ Both glucocorticoids and catecholamine release results in proliferation of myeloid-derived suppressor cells (MDSCs) present in the circulation. MDSCs are myeloid cell types that possess strong immunosuppressive activities. MDSCs interact with other immune cell types, including T cells, dendritic cells, macrophages, and natural killer cells (NK cells) to regulate their functions. MDSC release and suppress monocyte response and lymphopenia. In addition to release of MDSC, there is production of reactive oxygen species (ROS), which inhibit, decrease the number, or cause apoptosis of $\mathrm{T}$ cells and their function. This results in decreased production and release of cytokines and reduced lymphocyte activated killer cell cytotoxicity, resulting in impaired delayed hypersensitivity reaction..$^{13-15}$ All these mechanisms result in profound peripheral immune suppression ${ }^{10,13}(-$ Fig. 1).

Another mechanism involved is that the glucocorticoids and catecholamine released due to brain injury result in granulopoiesis, ${ }^{16}$ and immature neutrophils are released into the circulation. These immature neutrophils suppress T-cell function due to presence of CD10 and CD25 in them. ROS cause T-cell apoptosis. The net effect of these interplay of substances released results in lymphopenia and impaired hypersensitivity reaction and peripheral immune suppression.

Brain responds to peripheral immune response by three mechanisms via (1) the hypothalamic-pituitary-adrenal (HPA) axis, (2) sympathetic nervous system, and (3) parasympathetic nervous system. Through HPA axis, glucocorticoids inhibit proinflammatory cytokines and promote release of anti-inflammatory IL-4 and IL-10. They increase T-cell capacity to suppress macrophages, inhibit neutrophil phagocytosis, induce lymphocyte apoptosis, and downregulate antigen-containing monocytes..$^{10,13}$ Catecholamines released by sympathetic stimulation suppress $\beta$-adrenergic receptor-dependent neutrophil-generated ROS, proinflammatory cytokine production, and NK cells. They also enhance anti-inflammatory cytokines. Stimulation of $\beta$-adrenergic receptor influences differentiation of naive $\mathrm{T}$ cells to $\mathrm{T} 2$ and $\mathrm{T} 1$ cells. $\mathrm{T} 1$ and $\mathrm{T} 2$ cells are part of the immune system. T1 cells are proinflammatory and T2 are anti-inflammatory. When faced with a pathogenic attack, the body responds with a T1 response, which involves proinflammatory cytokines, along with other immune cells, allowing the body to kill invading organisms. Normally, the proinflammatory T1 response is balanced by anti-inflammatory T2 response. When the balance of T1/T2 cells favor anti-inflammatory response, it increases the susceptibility to infection, sepsis, and septic shock. ${ }^{13,14} \beta$-Adrenergic receptor antagonists have shown to reduce the risk of ischemia, enhance brain metabolism and oxygen consumption, and protect cardiovascular stability. ${ }^{17,18}$ In addition to sympathetic system, parasympathetic system stimulation also has been shown to decrease the systemic inflammatory response by decreasing the secretion of proinflammatory cytokines such as TNF- $\alpha$, IL-1, IL- 6 , and IL-18, without affecting the release of anti-inflammatory cytokine IL-10. This "cholinergic anti-inflammatory pathway" is more rapid and localized compared to diffuse anti-inflammatory property of the sympathetic system ${ }^{10,13,19}$ (- Fig. 2).

The anti-inflammatory pathway related to all the three above mechanisms results in increased peripheral immune suppression and susceptibility for infection, particularly pneumonia and septicemia. Toxicity as a result of septic shock, in the presence of hypotension, cardiovascular collapse, hemorrhage, and coagulation abnormalities is responsible for development of systemic organ failure such as acute renal failure, hepatic failure, and other systemic complication.

\section{Respiratory Complications}

Respiratory complications are the most common ECCs after severe head injury, with their incidence varying from $28 \%$ to $65 \%, 3,4,6-8,20-22$ The various complications are (1) neurogenic pulmonary edema (NPE), (2) ALI and ARDS, (3) pneumonia, and (4) lung collapse and atelectasis. Though these complications occur with definite symptoms and signs along with typical $\mathrm{X}$-ray finding, subclinical changes such as ventilation-perfusion abnormalities, increase in respiratory resistance, and impairment in muscle function cause hypoxia of varying degree. These respiratory changes occur early after brain injury and constitute independent risk factors of mortality and account for $>50 \%$ of patients dying due to brain damage. . $^{2-22}$

These respiratory complications develop as a result of "double hit" mechanisms..$^{21,22}$ First, the "blast injury" due to severe sympathetic response and release of catecholamines after brain injury, and the second "systemic inflammatory responses" in the brain with the release of pro- and antiinflammatory mediators released into the systemic circulation. 


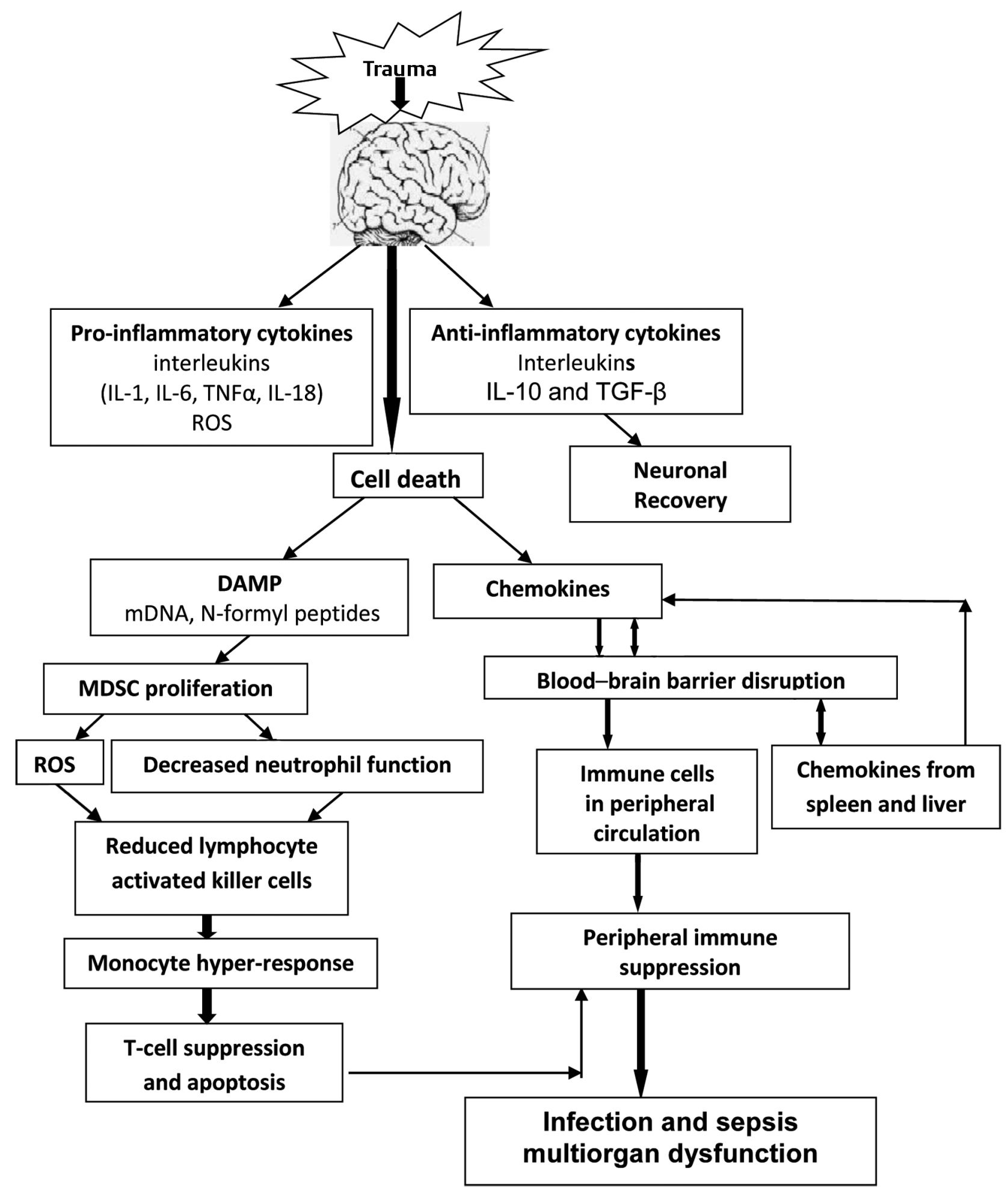

Fig. 1 Mechanism of immune suppression and multiorgan dysfunction due to traumatic brain injury. DAMP, nuclear and mitochondrial-associated molecular pattern; IL, interleukin; MDSC, myeloid-derived suppressor cell; ROS, reactive oxygen species; TGF- $\beta$, transforming growth factor- $\beta$; TNF- $\alpha$, tumor necrosis factor- $\alpha$.

\section{Pulmonary Edema}

Traumatic brain injury can result in abrupt increase in ICP leading to neuronal compression, ischemia, or damage, which is believed to give rise to an intense activation of the sympathetic nervous system and the release of catecholamines. The large amount of catecholamines released due to sympathetic storm caused by sudden and acute increase in ICP causes increase in intravascular pressure, systemic hypertension as well as pulmonary hypertension, and alveolar capillary membrane disruption. Extravasations of protei rich fluid from damaged alveolar-capillary membrane leads to 

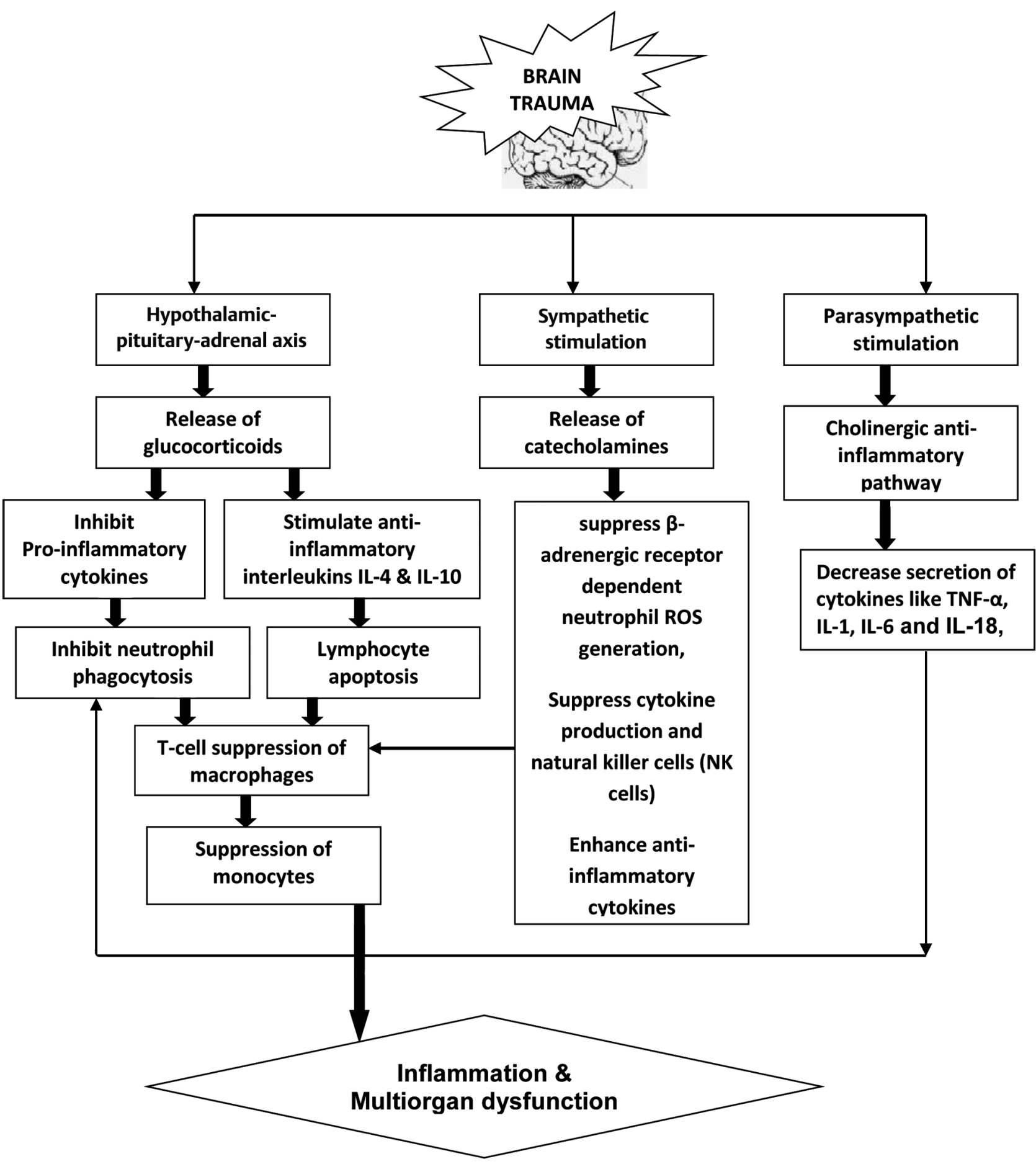

Fig. 2 Brain response to peripheral immune response. IL, interleukin; NK, natural killer; ROS, reactive oxygen species; TNF- $\alpha$, tumor necrosis factor- $\alpha$.

development of NPE. ${ }^{23,24}$ This entity was first described by Shahanan $^{25}$ in epileptic patients and mechanism described by Theodore and Robin in head injury. ${ }^{26}$ NPE differs from other types of pulmonary edema in that there is not only increase in capillary hydrostatic pressure but also increase in capillary permeability and accumulation of extravascular proteinaceous material (-Fig. 3). NPE generally occurs within 72 hours and is associated with high mortality (50\%). The severity of NPE is related to severity of TBI and severity of ICP increase. However, those who survive NPE recover very quickly. Experimental data from rat studies have shown that pretreatment with $\alpha$-adrenergic antagonist prevents the hypertensive response and decreases the severity of NPE and other respiratory complications. ${ }^{27}$

Acute Lung Injury and Acute Respiratory Distress Syndrome Brain injury leads to production of pro-inflammatory mediators. The microglia and astrocytes release tissue cytokines, 


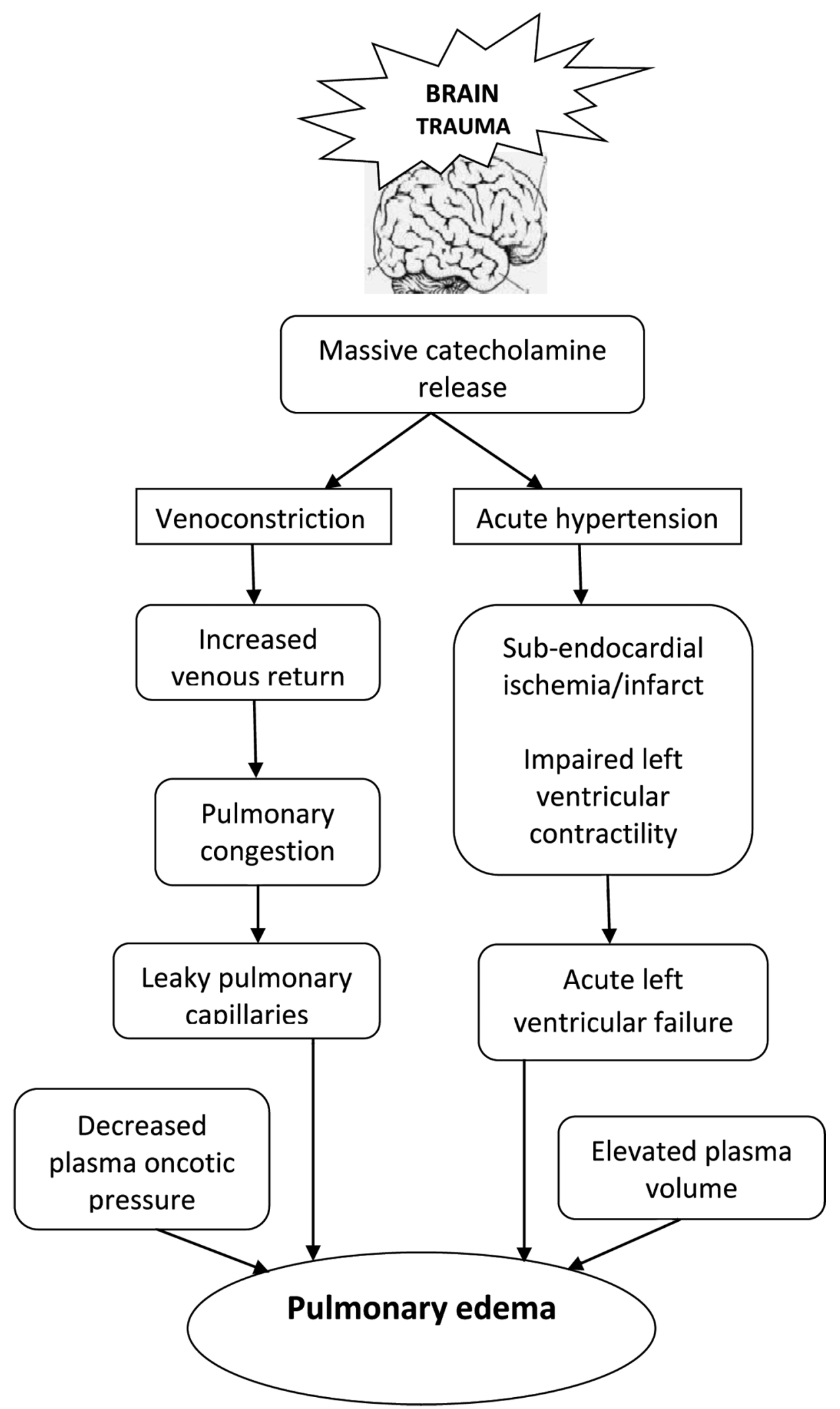

Fig. 3 Pathogenesis of neurogenic pulmonary edema.

IL-8, and other neurokinins. These inflammatory mediators cross disrupt the blood--brain barrier into the systemic circulation and activate inflammatory cascade in the lungs and other peripheral tissues. Neutrophils and macrophages migrate into the lungs and release pulmonary leukotrienes. In the lungs there is also upregulation of proinflammatory cytokines, substance-P, TNF- $\alpha$, IL- $\beta$, and IL-6. ${ }^{28,29}$ These kinins are also demonstrated in the bronchiolar lavage within 24 hours 
after acute TBI. ${ }^{30,31}$ Substance-P and neurokinins have been shown to cause bronchoconstriction, increase in vascular permeability, mucosal edema, and pulmonary edema.

The vagus and the parasympathetic system have a protective anti-inflammatory role in the lungs. Vagus nerve stimulation inhibits release of proinflammatory cytokines, TNF- $\alpha$, IL-1 IL-6, IL-8, and other inflammatory mediators. Medullary injury may initiate anti-parasympathetic activity adding to inflammatory response in the lung and exaggeration of ALI. Whatever may be the immune mechanism involved, brain injury produces ALI and ARDS. There is initial increase in airway resistance due to bronchoconstriction. ${ }^{30,31}$ Mucosal and alveolar edema results in hypoxia. Loss of surfactant results in airway closure, alveolar collapse, and atelectasis. Ventilation-perfusion mismatch and shunt result in impaired oxygenation. $\mathrm{PaO}_{2} / \mathrm{FiO}_{2}$ ratio of $<300$ is a good predictor of developing ARDS. The incidence of ARDS varies from 20 to $25 \%$ in isolated head injury and an independent predictor of mortality, poor neurological outcome, and is associated with longer ICU and hospital stay. Low GCS, presence of mass lesion with midline shift in the first scan, induced hypertension, need for vasopressors, young patients, presence of associated comorbidities such as hypertension, diabetes, chronic obstructive pulmonary disease (COPD), sepsis, cardiovascular, and renal and hematological dysfunctions are factors likely to be associated with development of ARDS. ${ }^{32,33}$ In addition, mechanical ventilation for ARDS with high tidal volume, high positive end-expiratory pressure (PEEP), recruitment maneuvers, and permissive hyperpnea itself may aggravate ARDS. ${ }^{34}$ Inadequate and improper ventilation strategies may result in aggravation of inflammation that could extend to other systemic organs. Most ARDS patients die of multiorgan failure and not of pulmonary dysfunction. ${ }^{35,36}$ ALI worsens the neurological outcome in patients with TBI. ${ }^{37}$ ARDS can lead to hippocampal injury, resulting in memory deficits and cognitive dysfunction. ${ }^{38}$

\section{Pneumonia}

Pneumonia generally presents around 5 to 7 days after TBI. The incidence of pneumonia varies between 45 and 70\%3,4 The nosocomial infection is the most common cause of pneumonia in head injury. Altered sensorium and obtunded pharyngeal and laryngeal reflexes predispose to aspiration. Older patients with diabetes, obesity, chronic pulmonary diseases, and more specifically use of barbiturate coma have been associated with development of pneumonia. ${ }^{39}$ Intubated patients on mechanical ventilation increase the risk of ventilator-associated pneumonia (VAP). Microaspiration, sedation, use of muscle relaxants, airway colonization, altered natural barrier due to the presence of endotracheal tube, and prior antibiotic usage increase the risk of VAP. Early initiation of oral or enteral feed and good oral care may reduce the risk of VAP. ${ }^{40}$ Blood transfusion and immune suppression due to brain injury further promote infection. Staphylococcus is the most common organism to cause pneumonia in ICU in these patients, though superadded gram-negative infection can complicate pneumonia. Fever, purulent secretions, and presence of infiltrates in the chest
X-ray suggest VAP. However, this should be differentiated from ALI, atelectasis, pulmonary contusion, pleural effusions, and pulmonary edema.

\section{Cardiovascular Complications}

Cardiovascular complications after severe head injury are common and often not attributed to TBI. The incidence varies from 45 to $65 \%{ }^{4-7}$ The various complications include (1) hypotension, (2) hypertension, (3) arrhythmias, (4) shock, and (5) neurogenic stunned myocardium.

The pathogenesis of cardiac injury is related to the sympathetic storm associated with TBI, due to central neuroendocrine axis, activation of adrenal gland stimulation, and release of massive amount of catecholamines. Increased ICP also causes sympathetic response. Autonomic dysfunction has been reported in TBI patients with damage to the insula and hypothalamus. ${ }^{41}$ This initiates complex and intense inflammatory response causing adverse reactions on the heart. ${ }^{41-44}$ Increased ICP also releases catecholamine in the sympathetic nerve endings of myocardium causing myocytic injury and cardiac dysfunction. Interestingly, there is no correlation between systemic catecholamine levels and the degree of myocardial injury, which, however, is directly related to the degree of catecholamine release at the sympathetic endings of heart. This increase in sympathetic activity results in opening of $\beta-1$ controlled calcium channels and depletion of ATP resulting in myocardial injury, which may be exaggerated by reperfusion. ${ }^{45,46}$ Myocardial injury results in mitochondrial dysfunction resulting in contraction-band-necrosis (CBN) characterized by focal myocytolysis and myofibrillar degeneration. These changes are more severe toward endocardium and progressively decrease toward epicardium. The severity of myocardial dysfunction is directly related to the degree of $\mathrm{CBN}$, which in turn depends on the severity of $\mathrm{TBI} .{ }^{47}$ The neuroinflammatory response to TBI resulting in myocytolysis and $C B N$ has been implicated in the pathogenesis of cardiac arrhythmias and myocardial dysfunction. Perfusion scans have shown that in addition to myocardial injury, catecholamines also produce vascular spasm of myocardial blood vessels resulting in regional perfusion abnormalities and ischemia. The clinical manifestations present in the form of hypotension and cardiac arrhythmias, ventricular dysfunction, and ischemic electrocardiogram (ECG) changes. Echocardiography may show regional wall motion abnormalities and systolic and diastolic dysfunction. ${ }^{48,49}$ This cardiac dysfunction after neurogenic injury is often referred to as "neurogenic stunned myocardium or stress cardiomyopathy" (Takotsubo's cardiomyopathy or broken heart syndrome $)^{42-44}$ (- Fig. 4).

The sympathetic response (and dysfunction of parasympathetic system) may continue for 48 to 72 hours. During this period, tachycardia, hypertension, and hyperdynamic status may occur. Frequently, bradycardia and hypertension due to Cushing's response may occur. Even though hypertension may improve cerebral perfusion, severe hypertension may cause heart failure. $\beta$-Blockers have shown to be useful in controlling the hypertension and improve the outcome. ${ }^{50}$ 


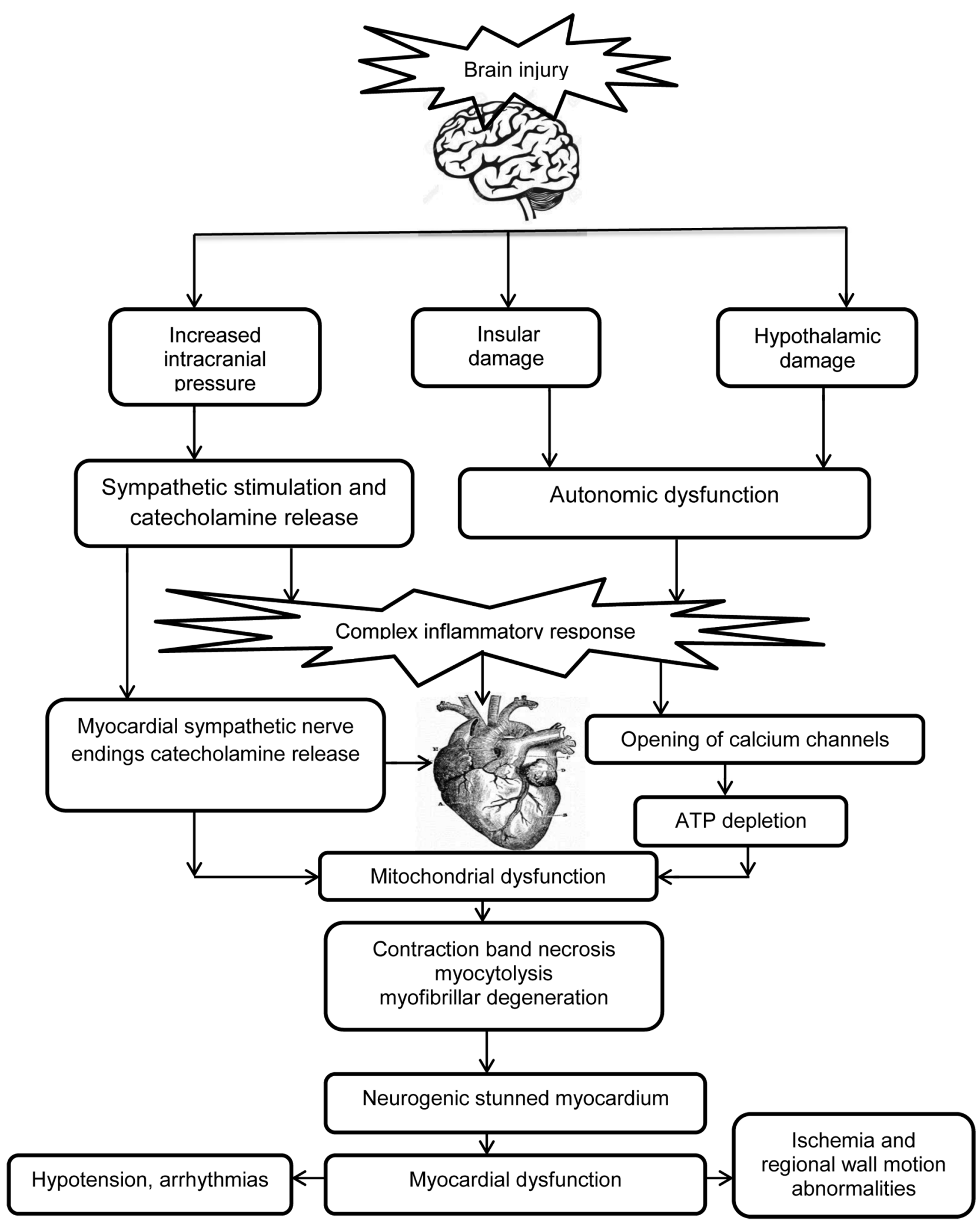

Fig. 4 Neurogenic stunned myocardium or stress cardiomyopathy (Takotsubo's cardiomyopathy or broken heart syndrome).

Once the surge of sympathetic stimulation decreases, the patient may develop hypotension. Hypotension in the immediate period of TBI is invariably due to extracranial causes such as hemorrhage or bleeding outside the cranium. However, hypotension not due to extracranial causes is invariably related to neurogenic hypotension, due to disruption of brainstem autonomic pathway, either due to direct injury or diffuse axonal injury, cerebral edema, medullary herniation, and spinal cord injury. Outcome in such cases is not favorable as compared with hypotension due to hemorrhagic shock, which, if treated with adequate blood transfusion, has better outcome. 
ECG changes reflect the changes due to neurogenic stunned myocardium. ${ }^{42}$ Most common changes are due to marked repolarization abnormality with QT prolongation and ST depression and T-wave changes occurring in 50 to $100 \%$ of patients. There could be bouts of polymorphic ventricular tachycardia, premature ventricular contractions, and wide QRS complexes. ECG changes may mimic acute coronary ischemia and may be difficult to differentiate from the myocardial infarction. Neurogenic ECG changes are generally asymptomatic. Cardiac enzymes changes may help in identifying ischemic or infarct events..$^{20,42,43}$ Repolarization changes revert with improvement in the neurological status. Sudden cardiac arrest has been noted to occur in patients with prolonged QTc.

Cardiac arrhythmias are not very common, occurring in about $25 \%$ of patients. Tachycardia, premature atrial or ventricular contraction, or atrial fibrillation may occur. However, most neurogenic arrhythmias are benign and disappear with improvement in neurological condition. However, prolonged QTc, torsades de pointes, and ventricular fibrillation occasionally occur in patients with insular lesions and may be life threatening.

\section{Coagulation Disorders}

Acute traumatic coagulopathy (ATC) is a well-known entity after severe trauma or crush injuries. The incidence of ATC in head injury varies depending on the definition of what constitutes coagulopathy. Many authors have used different parameters to define ATC, such as international normalized ratio (INR), platelet count, prothrombin time (PT), activated partial thromboplastin time (aPTT), fibrinogen level, disseminated intravascular coagulation (DIC) score, modified coagulopathy score, and $\alpha_{2}$-plasmin inhibitor value. ${ }^{51}$ In a meta-analysis by Epstein et al, depending on the definition, the incidence varies from 7 to $83 \%$. However, pooled incidence was $35.2 \%$. The mortality rate in ATC was 17 to $86 \%$ in different studies. ${ }^{51}$

The reason why ATC occurs in TBI is because the brain contains the highest concentration of tissue factor (TF) or tissue thromboplastin in the body. Hence, any injury to the brain releases TF into the circulation initiating coagulation cascade. The process of fibrin formation may deplete coagulation factors and platelets, resulting in hypercoagulable state. Fibrinolytic activity may also initiate diffuse intravascular coagulation (DIC). ATC can cause bleeding and intracerebral hematoma formation and carry very high mortality. ${ }^{52,53}$

Traumatic coagulopathy is the result of imbalance between pro- and anticoagulants. With brain tissue injury, there is liberation of TF, which initiates the coagulation cascade. With endothelial damage, platelets, and activated factors, thrombin is formed by extrinsic pathway. The thrombomodulin (TM) present in the damaged endothelium combines with thrombin to form a complex, after which no thrombin is available for cleavage of fibrinogen. This complex activates protein $\mathrm{C}$ that inhibits cofactors V and VIII inhibiting further thrombin formation. ${ }^{54,55}$ When hypotension is present, injury to endothelium releases tissue plasminogen activator (tPA) that splits plasminogen and activates fibrinolysis. Activated protein $\mathrm{C}$ consumes plasminogen activator inhibitor (PAI-1) that leads to increased tPA activity and hyperfibrinolysis. ${ }^{54,55}$

Acute coagulopathy due to brain injury is associated with platelet dysfunction. ${ }^{56}$ Severity of platelet dysfunction is related to severity of brain injury, even though the platelet count may not be reduced. Davis et $\mathrm{al}^{56}$ evaluated the platelet function in patients with isolated head injury by thromboelastography with platelet mapping (TEG-PM). The study showed no correlation between platelet count and platelet dysfunction as analyzed by TEG-PM. Hence platelet count per se may not be a good indicator of coagulopathy. The exact mechanism of platelet dysfunction is not clear. However, platelet ADP receptor inhibition has been shown to directly correlate with the low GCS, severity of TBI, and mortality. ${ }^{56}$

Coagulation disorders cause not only bleeding, blood loss, and intra- and extracranial hemorrhages but also shock. Widespread coagulation cascade results in systemic inflammatory response and sepsis. Immunological reaction to blood transfusion might also add to the inflammatory response. Sepsis and septic shock may result in organ dysfunction and multiorgan failure. ${ }^{53}$

\section{Risk Factors for Acute Traumatic Coagulopathy in Head Injury}

In a study by Abdelmalik et $\mathrm{al}^{52}$ on ATC in isolated head injury, highest incidence of ATC was noted on day 2, both with mild and severe head injuries. However, ATC persisted for greater number of days with severe TBI as compared with mild injury. Other factors that were associated with ATC were (1) hyperthermia temperature $>38.5^{\circ} \mathrm{C}$, (2) hypothermia temperature $<35^{\circ} \mathrm{C}$, (3) hypotension with systolic blood pressure < $90 \mathrm{~mm} \mathrm{Hg}$, (4) oxygen $\mathrm{PO}_{2}<60 \mathrm{~mm} \mathrm{Hg}$, (5) GCS $<8$, and (6) lactate level $>4.0 \mathrm{mM} / \mathrm{L}$. All patients had high INR and PT. There was higher incidence of intracerebral hemorrhages and hematoma formation, with subarachnoid hemorrhages and midline shift. The length of hospital stay, incidence of deep vein thrombosis (DVT), and mortality was higher in ATC patients. In those patients who got discharged, long-term functional outcomes in ATC patients were not encouraging and most patients had significant moderate to severe cognitive dysfunction. ${ }^{52}$ As reported by Epstein et al in their meta-analysis, ${ }^{51}$ mortality was highest in patients who developed ATC within first 24 hours. Increasing age, low GCS on admission, male patients, pupillary reflex abnormalities, and cerebral hypoperfusion in patients with ATC, were associated with poor outcome. In a large multicenter population-based trauma registry-based study, Wafaisade et $\mathrm{al}^{57}$ analyzed adult patients with isolated blunt TBI with intracranial AIS $\geq 3$ and extracranial AIS score $<3$, for acute posttraumatic coagulopathy upon arrival at emergency room (ER). The incidence of acute coagulopathy in isolated TBI was $22.7 \%$. Overall hospital mortality was $50 \%$ in patients with coagulopathy. GCS $<8$, presence of hypotension at the scene or ER, fluid resuscitation volume of $>2 \mathrm{~L}$, and age $>75$ years were all independent factors of mortality. Other significant complications between ATC and non-ATC patients were single organ failure (60.7\% vs. $38.7 \%)$, multiorgan failure $(35.7 \%$ 
vs. $19.4 \%$ ), intubation-free days ( $11 \pm 13$ vs. $20 \pm 12$ ), hospital length of stay ( $15 \pm 22$ vs. $18 \pm 20$ ), discharge from hospital (31.8\% vs. $62.3 \%), 24$-hour mortality ( $26.1 \%$ vs. $4.4 \%$ ), and overall hospital mortality (50.4\% vs. $17.3 \%$ ), thus suggesting that patients with ATC develop more complications resulting in mortality.

\section{Endocrine Dysfunction}

Endocrine dysfunction has been reported after moderate to severe head injury in the first week after injury. ${ }^{10}$ The incidence of acute dysfunction of both anterior and posterior pituitary is about 4 to $8 \%$. However, their presentation is often subclinical and may be missed clinically. These may present in the form of adrenal dysfunction, thyroid dysfunction, and antidiuretic hormone dysfunction. However, gonadotrophins and growth hormone abnormalities are also reported in recovery and late phase of head injury. ${ }^{10}$ Pituitary hematoma due to basilar fracture of the skull, raised ICP, hypoxia, hypotension, and cytokine inflammatory mediators released with TBI has been found to be associated with endocrine dysfunction. Treatment with phenytoin and use of etomidate may also precipitate pituitary dysfunction. ${ }^{10}$

Late-onset endocrine dysfunction after TBI may present between 6 months and 5 years after recovery from TBI. This endocrine dysfunction occurs after moderate to severe head injury; however, it is not commonly recognized in the acute phase but becomes evident in the chronic stage beyond 6 months. ${ }^{10}$ In a study by Yang et $a l^{58}$ endocrine dysfunction was noticed in $0.4 \%$, as compared with $0.3 \%$ in non-TBI patients at 1-year follow-up, and the incidence increased to $2 \%$ over 5 -year follow-up.

The common endocrine dysfunctions during early phase of TBI are (1) anterior and posterior pituitary dysfunction occurring in about 4 to $8 \%$, (2) adrenal dysfunction, (3) thyroid dysfunction, and (4) antidiuretic hormone dysfunction. However, gonadotrophins and growth hormone abnormalities are also reported during recovery and in the late phase of head injury..$^{10}$ In a study by Yang et $a 1,{ }^{58}$ in a follow-up of 31,389 patients with head injuries for 5 years, endocrine dysfunction was noticed in $0.4 \%$ as compared with $0.3 \%$ in non-TBI patients at 1-year follow-up, and the incidence increased to $2 \%$ over 5 -year follow-up. Direct injury to the pituitary gland due to base of the skull fracture, disruption of the infundibulum, and interruption in blood supply due to damage to hypophyseal blood vessels may result in pituitary hemorrhage or infarction, and hypothalamic damage, and has been responsible for hypopituitarism.

Adrenal insufficiency in the early phase may present as hemodynamic instability with inability to maintain blood pressure, in spite of adequate blood volume due to low systemic vascular resistance. Other manifestations are poor response to vasopressors, hypoglycemia, and hyponatremia. Serum cortisol levels are found to be low and are diagnostic of hypoadrenalism. Acute hypoadrenalism has been found to be more common in patients on mechanical ventilation. Recurrent infections, hyponatremia, and hypotension are common in chronic phase. ${ }^{10,58}$
Hypothyroidism due to pituitary cause has been reported in 17 to $24 \%$ of patients on days 1 and 2, after TBI. The peripheral conversion of thyroxine $\left(\mathrm{T}_{4}\right)$ to triiodothyronine $\left(\mathrm{T}_{3}\right)$ is impaired, and abnormal form of T3 known as reverse T3 (rT3) has been found to be elevated. rT3 is a biologically inactive metabolite of $\mathrm{T}_{4}$ formed by selective de-iodination. This condition is known as "euthyroid sick syndrome" due to dysregulation of thyrotropic feedback control, wherein the levels of $\mathrm{T}_{3}$ and/or $\mathrm{T}_{4}$ are abnormal, but the thyroid gland does not appear to be dysfunctional..$^{59}$ Clinical features of hypothyroidism may not be obvious. Low levels of thyroidstimulating hormone (TSH) (due to pituitary lesion) and absence of TSH-releasing factor may result in prolonged coma and poor outcome. Chronic phase may present with typical features of hypothyroidism with low TSH levels.

Endocrine dysfunction after TBI occurring during late phase may present with vague symptoms such as nonspecific weakness, memory loss, attention problems, and others. These patients need to be evaluated with serum cortisol level, thyroid hormone levels, and other investigations.

\section{Electrolyte Disturbances}

Electrolyte disturbances are more common and present from day 2 of TBI. Piek et al and others have shown that electrolyte disturbances occur in more than $59 \%$ of patients with TBI.-6,10 Hyponatremia is the most common abnormality reported. This is due to syndrome of inappropriate ADH secretion (SIADH) and cerebral salt-wasting syndrome (CSWS). Both these conditions cause hyponatremia. However, the distinction between these two syndromes should be recognized, as the treatment differs. SIADH occurs due to excess ADH release, resulting in excess water retention, hyponatremia, and decreased serum osmolarity due to dilution and elevated urine osmolarity due to excretion of sodium into the urine. The blood volume in mild cases may be normal, but in severe cases, it may show increase. Hence SIADH is treated with water restriction. CSWS syndrome occurs due to elevated natriuretic factor and results in decreased blood volume and hyponatremia. Treatment of CSWS is by volume replacement with sodium containing fluid.

Injury to the posterior pituitary gland or hypothalamus may result in ADH deficiency producing hypernatremia and diabetes insipidus (DI). The incidence of DI is about 14.7 to $25 \%{ }^{60}$ Risk factors for DI are severe TBI with low GCS and cerebral edema. Mild DI may be treated with fluid replacement, but severe DI may require vasopressin. DI was found to be one of the independent risk factors for mortality. ADH secretion disturbances are generally transient and recover within few days. Other electrolyte disturbances such as potassium and magnesium changes are more related to treatment.

\section{Other Systems}

\section{Gastrointestinal System}

As in any stressful conditions, TBI can result in acute gastric ulcers (Cushing's ulcer). They are observed endoscopically within 24 hours after TBI and may cause significant 
hematemesis. Injury to the diencephalon and brainstem has been shown to increase gastrin and gastric acid output. TBI increases intestinal TNF- $\alpha$, interleukins, and intercellular adhesion molecules. These immune mediators disrupt the intestinal barrier and increase intestinal permeability and endotoxin absorption. Ileus may occur due to endotoxin absorption. Progesterone has been shown to reduce intestinal inflammation. ${ }^{61}$

\section{Metabolism}

TBI induces hypermetabolic state with increase in caloric requirement by more than two to three times. Changes in the amino acids have been correlated with ICP and cerebral oxygen consumption. Elevated plasma leucine and isoleucine amino acids were associated with increased ICP and increased cerebral metabolic rate of oxygen $\left(\mathrm{CMRO}_{2}\right)$. However, increased phenylalanine level was associated with decreased ICP and $\mathrm{CMRO}_{2}{ }^{10,62}$ Feedings should be initiated within 72 years, and enteral feeding should be preferred as this would maintain gut integrity and decrease infection. Increased stress response leads to hyperglycemia that is associated with increased morbidity and mortality. ${ }^{10}$

\section{Temperature Regulation}

Patients with head injury may develop fever, which may be infectious or noninfectious. TBI is often associated with impairment of body temperature. Many studies have shown that the brain temperature tends to be higher than the core body temperature by nearly $1^{\circ} \mathrm{C} .{ }^{62}$ This "neurogenic fever" has been shown to occur in 4 to $37 \%$ of patients with diffuse axonal injury and also in patients with frontal lobe injury or with base of skull fractures. ${ }^{63}$ This noninfectious fever has been attributed to injury to hypothalamic temperatureregulating center. Autopsy studies have shown incidence of hypothalamic injury in $42 \%$ of patients dying of severe brain injury. ${ }^{64}$ Neurogenic fever may also occur due to posttraumatic changes in brain metabolism due to hyperglycolysis, increase in cerebral blood flow (CBF), and also due to inflammatory response in the brain with the release of interleukins, particularly within 24 to 48 hours. Presence of blood in cerebrospinal fluid (CSF) or intraventricular bleed may lead to fever. Hyperthermia releases free radicals and excitatory amino acids and causes skeletal muscle proteolysis. Hyperthermia increases cerebral metabolic rate and blood-brain barrier breakdown. Sacho et al have shown that brain temperature more than $38^{\circ} \mathrm{C}$ and less than $36.5^{\circ} \mathrm{C}$ is associated with higher mortality. ${ }^{65}$ Spontaneous hypothermia may occur in patients exposed to low ICU temperature unless adequate measures are taken to warm the patient. Hypothermia decreases both cellular and humeral immunity and increases the risk of infection. ${ }^{66}$ Fall in brain temperature as compared with body temperature is associated with poor prognosis. Hypothermia occurs when brain death occurs.

\section{Conclusion}

The neurogenic inflammatory response to TBI releases cascades of inflammatory materials that cross the disrupted blood-brain barrier, ultimately causing suppression of peripheral immunity. Though these inflammatory mediators are supposed to be responsible for reparative process of the organs, they can cause cellular apoptosis. The materials released by apoptotic cells result in cascade of inflammation, infection, and sepsis. Septic shock may result in acute multiorgan failure. Hence, even though head injury may present as an isolated injury, if severe enough to cause disruption of bloodbrain barrier, it may result in systemic organ dysfunction.

\section{Conflict of Interest}

None declared.

\section{Acknowledgments}

I would like to acknowledge the assistance of Dr. Anita Pramod in correcting the manuscript. I would also like to acknowledge the support provided by the colleagues of the Department of Anesthesiology, Manipal Hospital, for preparation of this manuscript.

\section{References}

1 Shekhar C, Gupta LN, Premsagar IC, Sinha M, Kishore J. An epidemiological study of traumatic brain injury cases in a trauma centre of New Delhi (India). J Emerg Trauma Shock 2015;8(3):131-139

2 Kamal VK, Agrawal D, Pandey RM. Epidemiology, clinical characteristics and outcomes of traumatic brain injury: evidences from integrated level 1 trauma center in India. J Neurosci Rural Pract 2016;7(4):515-525

3 Piek J, Chesnut RM, Marshall LF, et al. Extracranial complications of severe head injury. J Neurosurg 1992;77(6):901-907

4 Schirmer-Mikalsen K, Vik A, Gisvold SE, Skandsen T, Hynne H, Klepstad P. Severe head injury: control of physiological variables, organ failure and complications in the intensive care unit. Acta Anaesthesiol Scand 2007;51(9):1194-1201

5 Corral L, Javierre CF, Ventura JL, Marcos P, Herrero JI, Mañez R. Impact of non-neurological complications in severe traumatic brain injury outcome. Crit Care 2012;16(2):R44

6 Zygun DA, Kortbeek JB, Fick GH, Laupland KB, Doig CJ. Non-neurologic organ dysfunction in severe traumatic brain injury. Crit Care Med 2005;33(3):654-660

7 Zygun DA, Doig CJ, Gupta AK, et al. Non-neurological organ dysfunction in neurocritical care. J Crit Care 2003;18(4):238-244

8 Zygun D. Non-neurological organ dysfunction in neurocritical care: impact on outcome and etiological considerations. Curr Opin Crit Care 2005;11(2):139-143

9 Zygun D, Berthiaume L, Laupland K, Kortbeek J, Doig C. SOFA is superior to MOD score for the determination of non-neurologic organ dysfunction in patients with severe traumatic brain injury: a cohort study. Crit Care 2006;10(4):R115

10 Gaddam SS, Buell T, Robertson C. Systemic manifestations of traumatic brain injury. In: Jordan G, Andres S, eds. Handbook of Clinical Neurology. Elsevier;2015;127:205-218

11 Baguley IJ, Perkes IE, Fernandez-Ortega JF, Rabinstein AA, Dolce G, Hendricks HT; Consensus Working Group. Paroxysmal sympathetic hyperactivity after acquired brain injury: 
consensus on conceptual definition, nomenclature, and diagnostic criteria. J Neurotrauma 2014;31(17):1515-1520

12 Meyfroidt G, Baguley IJ, Menon DK. Paroxysmal sympathetic hyperactivity: the storm after acute brain injury. Lancet Neurol 2017;16(9):721-729

13 Hazeldine J, Lord JM, Belli A. Traumatic brain injury and peripheral immune suppression: primer and prospectus [review]. Front Neurol 2015;6:235

14 Wolach B, Sazbon L, Gavrieli R, Broda A, Schlesinger M. Early immunological defects in comatose patients after acute brain injury. J Neurosurg 2001;94(5):706-711

15 Smrcka M, Mrlian A, Klabusay M. Immune system status in the patients after severe brain injury. Bratisl Lek Listy 2005;106(3):144-146

16 Subramanian A, Agrawal D, Pandey RM, Nimiya M, Albert V. The leukocyte count, immature granulocyte count and immediate outcome in head injury patients. In: Agrawal A, ed. Brain Injury-Pathogenesis, Monitoring, Recovery and Management;2012. Open access. Available at:https://www.intechopen. com/books/. Accessed June 17, 2019

17 Inaba K, Teixeira PG, David JS, et al. Beta-blockers in isolated blunt head injury. J Am Coll Surg 2008;206(3):432-438

18 Meyer JS, Miyakawa Y, Welch KM, et al. Influence of adrenergic receptor blockade on circulatory and metabolic effects of disordered neurotransmitter function in stroke patients. Stroke 1976;7(2):158-167

19 Borovikova LV, Ivanova S, Zhang M, et al. Vagus nerve stimulation attenuates the systemic inflammatory response to endotoxin. Nature 2002;420:853-859

20 Lim HB, Smith M. Systemic complications after head injury: a clinical review. Anaesthesia 2007;62(5):474-482

21 Alvarez JML, Quevedo OP, Furelos LR, et al. Pulmonary complications in patients with brain injury. Pulm Res Med Open J 2015;2(1):69-74

22 Koutsoukou A, Katsiari M, Orfanos SE, et al. Respiratory mechanics in brain injury: a review. World J Crit Care Med 2016;5(1):65-73

23 Smith WS, Matthay MA. Evidence for a hydrostatic mechanism in human neurogenic pulmonary edema. Chest 1997;111(5):1326-1333

24 McClellan MD, Dauber IM, Weil JV. Elevated intracranial pressure increases pulmonary vascular permeability to protein. J Appl Physiol (1985) 1989;67(3):1185-1191

25 Shanahan WT. Pulmonary edema in epilepsy. NY Med 1908;87:54-56

26 Theodore J, Robin ED. Pathogenesis of neurogenic pulmonary oedema. Lancet 1975;2(7938):749-751

27 Avlonitis VS, Wigfield CH, Kirby JA, Dark JH. The hemodynamic mechanisms of lung injury and systemic inflammatory response following brain death in the transplant donor. Am J Transplant 2005;5(4 Pt 1):684-693

28 Kalsotra A, Zhao J, Anakk S, Dash PK, Strobel HW. Brain trauma leads to enhanced lung inflammation and injury: evidence for role of P4504Fs in resolution. J Cereb Blood Flow Metab 2007;27(5):963-974

29 Skrabal CA, Thompson LO, Potapov EV, et al. Organ-specific regulation of pro-inflammatory molecules in heart, lung, and kidney following brain death. J Surg Res 2005;123(1):118-125

30 Fisher AJ, Donnelly SC, Hirani N, et al. Enhanced pulmonary inflammation in organ donors following fatal non-traumatic brain injury. Lancet 1999;353(9162) :1412-1413

31 Fisher AJ, Donnelly SC, Hirani N, et al. Elevated levels of interleukin-8 in donor lungs is associated with early graft failure after lung transplantation. Am J Respir Crit Care Med 2001;163(1):259-265

32 Contant CF, Valadka AB, Gopinath SP, Hannay HJ, Robertson CS. Adult respiratory distress syndrome: a complication of induced hypertension after severe head injury. J Neurosurg 2001;95(4):560-568

33 Bratton SL, Davis RL. Acute lung injury in isolated traumatic brain injury. Neurosurgery 1997;40(4):707-712, discussion712

34 Mascia L, Zavala E, Bosma K, et al; Brain IT group. High tidal volume is associated with the development of acute lung injury after severe brain injury: an international observational study. Crit Care Med 2007;35(8):1815-1820

35 Hegeman MA, Hennus MP, Heijnen CJ, et al. Ventilator-induced endothelial activation and inflammation in the lung and distal organs. Crit Care 2009;13(6):R182

36 Slutsky AS, Tremblay LN. Multiple system organ failure. Is mechanical ventilation a contributing factor? Am J Respir Crit Care Med 1998;157(6 Pt 1):1721-1725

37 Holland MC, Mackersie RC, Morabito D, et al. The development of acute lung injury is associated with worse neurologic outcome in patients with severe traumatic brain injury. J Trauma 2003;55(1):106-111

38 Hopkins RO, Brett S. Chronic neurocognitive effects of critical illness. Curr Opin Crit Care 2005;11(4):369-375

39 Bronchard R, Albaladejo P, Brezac G, et al. Early onset pneumonia: risk factors and consequences in head trauma patients. Anesthesiology 2004;100(2):234-239

40 Kollef MH, Morrow LE, Niederman MS, et al. Clinical characteristics and treatment patterns among patients with ventilator-associated pneumonia. Chest 2006;129(5):1210-1218

41 Krishnamoorthy V, Mackensen GB, Gibbons EF, Vavilala MS. Cardiac dysfunction after neurologic injury: what do we know and where are we going? Chest 2016;149(5):1325-1331

42 Gregory T, Smith M. Cardiovascular complications of brain injury. Contin Educ Anaesth Crit Care Pain 2012;12(2):67-71

43 Manikandan S. Heart in the brain injured. J Neuroanaesth Crit Care 2016;3(Suppl S1):12-15

44 Nguyen H, Zaroff JG. Neurogenic stunned myocardium. Curr Neurol Neurosci Rep 2009;9(6):486-491

45 Samuels MA. The brain-heart connection. Circulation 2007; 116(1):77-84

46 Greenhoot JH, Reichenbach DD. Cardiac injury and subarachnoid hemorrhage. A clinical, pathological, and physiological correlation. J Neurosurg 1969;30(5):521-531

47 Tung P, Kopelnik A, Banki N, et al. Predictors of neurocardiogenic injury after subarachnoid hemorrhage. Stroke 2004;35(2):548-551

48 Taggart P, Critchley H, Lambiase PD. Heart-brain interactions in cardiac arrhythmia. Heart 2011;97(9):698-708

49 Frangiskakis JM, Hravnak M, Crago EA, et al. Ventricular arrhythmia risk after subarachnoid hemorrhage. Neurocrit Care 2009;10(3):287-294

50 Cotton BA, Snodgrass KB, Fleming SB, et al. Beta-blocker exposure is associated with improved survival after severe traumatic brain injury. J Trauma 2007;62(1):26-33, discussion 33-35

51 Epstein DS, Mitra B, O’Reilly G, Rosenfeld JV, Cameron PA. Acute traumatic coagulopathy in the setting of isolated traumatic brain injury: a systematic review and meta-analysis. Injury 2014;45(5):819-824

52 Abdelmalik PA, Boorman DW, Tracy J, Jallo J, Rincon F. Acute traumatic coagulopathy accompanying isolated traumatic brain injury is associated with worse long-term functional and cognitive outcomes. Neurocrit Care 2016;24(3):361-370

53 Harhangi BS, Kompanje EJO, Leebeek FWG, Maas AI. Coagulation disorders after traumatic brain injury. Acta Neurochir (Wien) 2008;150(2):165-175, discussion 175

54 Brohi K, Cohen MJ, Ganter MT, Matthay MA, Mackersie RC, Pittet JF. Acute traumatic coagulopathy: initiated by hypoperfusion: modulated through the protein C pathway? Ann Surg 2007;245(5):812-818 
55 Frith D, Brohi K. The pathophysiology of trauma-induced coagulopathy. Curr Opin Crit Care 2012;18(6):631-636

56 Davis PK, Musunuru H, Walsh M, et al. Platelet dysfunction is an early marker for traumatic brain injury-induced coagulopathy. Neurocrit Care 2013;18(2):201-208

57 Wafaisade A, Lefering R, Tjardes T, et al; Trauma Registry of DGU. Acute coagulopathy in isolated blunt traumatic brain injury. Neurocrit Care 2010;12(2):211-219

58 Yang WH, Chen PC, Wang TC, Kuo TY, Cheng CY, Yang YH. Endocrine dysfunction following traumatic brain injury: a 5-year follow-up nationwide-based study. Sci Rep 2016;6:32987

59 Woolf PD, Lee LA, Hamil RW, et al. Thyroid test abnormalities in traumatic brain injury: correlation with neurological impairment and sympathetic system. Am J Med 1988;84:201-208

60 Agha A, Thornton E, O'Kelly P, Tormey W, Phillips J, Thompson CJ. Posterior pituitary dysfunction after traumatic brain injury. J Clin Endocrinol Metab 2004b;89(12):5987-5992

61 Jin W, Wamg H, Ji Y, et al. Increased intestinal inflammatory response and gut barrier dysfunction in Nrf-2 deficient mice after traumatic brain injury. Cytokine 2008;44:134-140
62 Rumana CS, Gopinath SP, Uzura M, Valadka AB, Robertson CS. Brain temperature exceeds systemic temperature in head-injured patients. Crit Care Med 1998;26(3):562-567

63 Thompson HJ, Pinto-Martin J, Bullock MR. Neurogenic fever after traumatic brain injury: an epidemiological study.J Neurol Neurosurg Psychiatry 2003;74(5):614-619

64 Crompton MR. Hypothalamic lesions following closed head injury. Brain 1971;94(1):165-172

65 Sacho RH, Vail A, Rainey T, King AT, Childs C. The effect of spontaneous alterations in brain temperature on outcome: a prospective observational cohort study in patients with severe traumatic brain injury. J Neurotrauma 2010;27(12):2157-2164

66 Henderson WR, Dhingra VK, Chittock DR, Fenwick JC, Ronco JJ. Hypothermia in the management of traumatic brain injury. A systematic review and meta-analysis. Intensive Care Med 2003;29(10):1637-1644 